\title{
Characterization of 16 MegaPixel CMOS Detector for TEM by Evaluating Single Events of Primary Electrons
}

\author{
Ghadimi R., Daberkow I., Kofler C., Sparlinek P., and Tietz H.R. \\ TVIPS GmbH, Eremitenweg 1, 82131 Gauting, Germany
}

Within the last two decades, CCD cameras (Charge Coupled Device) turned out to be an important tool for image recording in TEM. In 2008 and 2009 TVIPS introduced 16 and 64 MegaPixel cameras based on CMOS technology (Complementary Metal Oxide Semiconductor). Both cameras have a large pixel size of $15.6 \mu \mathrm{m}$, a fiber-optic coupled scintillator and a special readout mode CDS (Correlated Double Sampling) that leads to an extreme high sensitivity allowing a clear detection of single primary electrons.

Standard parameters are used to determine the main camera characteristics. These are the lateral resolution MTF (Modulation Transfer Function), the sensitivity vs. readout noise (mean signal for single primary electrons with respect to the readout noise), and dynamic range (ratio of maximum to noise signal). The resolution-dependent DQE (Detection Quantum Efficiency) combines the resolution, sensitivity, and noise characterization.

The determination of the MTF is difficult in general, because special equipment is needed, e.g. a sharp edge positioned directly above the scintillator. Another problem is the insufficient opaqueness of the edge especially for higher electron energies. We present a method that evaluates the signal of single electron events at extreme low intensities. This has several advantages:

1) The lateral resolution can easily be determined by averaging the single events. The result is the PSF (Point Spread Function). The Fourier transform of the PSF delivers the MTF.

2) Without any current density calibration, the integrated signal of the accumulated single events is a measurand of the sensitivity.

3) The evaluation method can be applied to any other electron detector with sufficient sensitivity as well. This method could be used as a standard for a careful side-by-side comparison of different detectors that is not influenced by individual shape, geometry or material of the edge, and by different mathematical algorithms.

The method has been applied to our CMOS TemCam-F416 camera ( $4 \mathrm{k} \times 4 \mathrm{k}, 15.6 \mu \mathrm{m}$ pixel). Using the High Resolution (HR) scintillator it has a mean response of about 70 counts per single $200 \mathrm{kV}$ primary electron. Taking into account a readout noise level of 5 counts, this leads to a clear detection of single electron events with an average signal-to-noise ratio of 14:1 (refer Fig. 1).

Fig. 2 shows a histogram of 11670 events that have been selected by a dedicated search algorithm. It takes into account a lower threshold value and a Kernel size of 7x7. Each event is expanded with a 20x20 cubic-spline interpolation. These events are individually aligned using the center of gravity and then averaged. To determine the sensitivity all signals above the noise level have been summed up and displayed in the histogram. Fig. 3 shows the 2-dimensional PSF (distribution of the averaged events) and Fig. 4 its rotational average. Fig. 5 shows the corresponding MTF. Please notice this 
MTF is already corrected for aliasing effects, and the Nyquist frequency is defined as reciprocal of twice the pixel spacing.
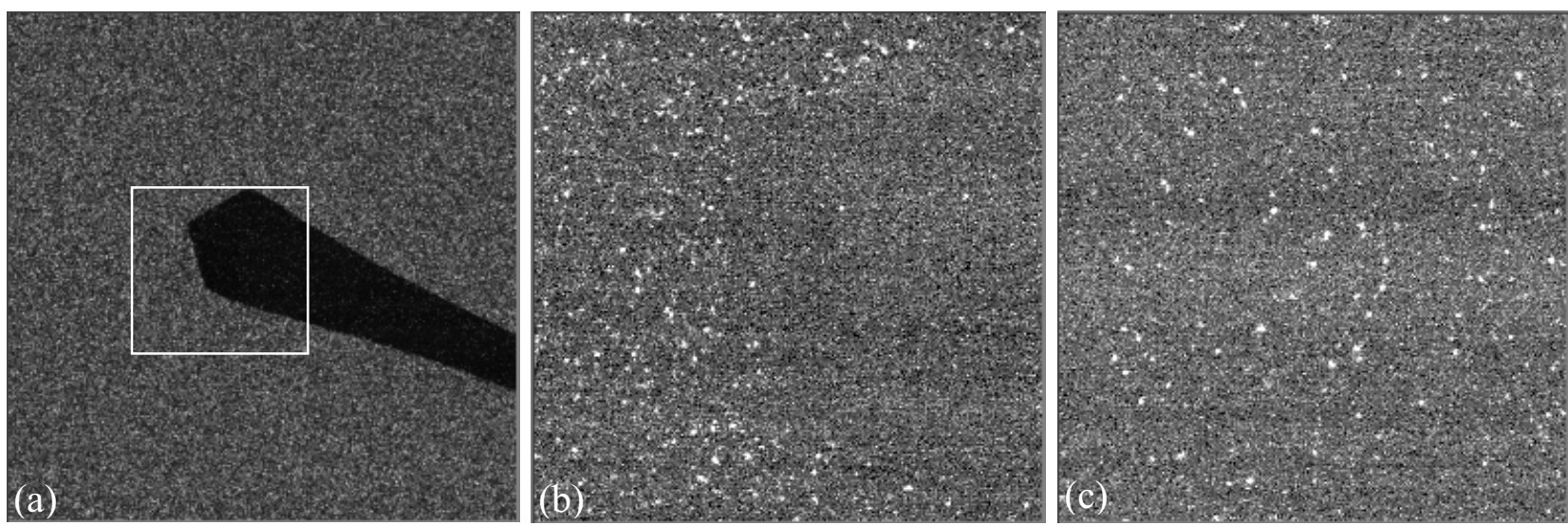

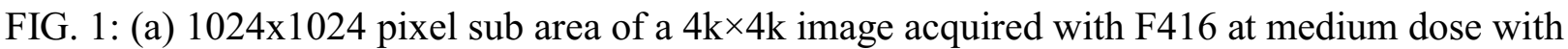
the beamstop in the center position; (b) shows a $256 \times 256$ pixel sub area of (a) at a very low dose; (c) shows a typical sub area of the image that has been used for evaluation. Please note single electron events are clearly visible.

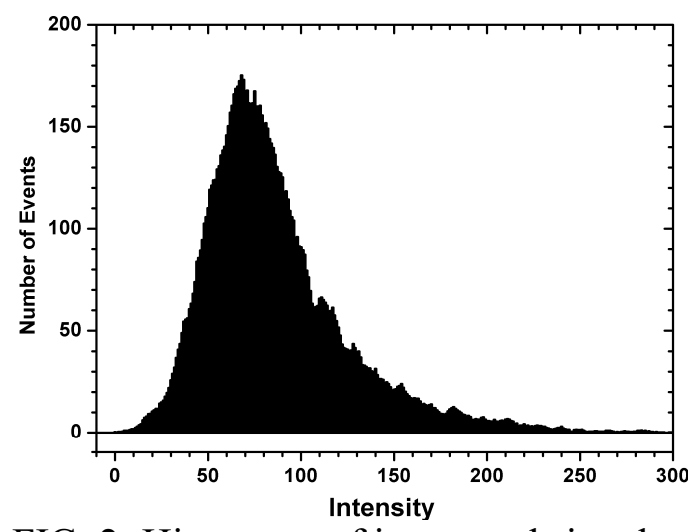

FIG. 2: Histogram of integrated signals

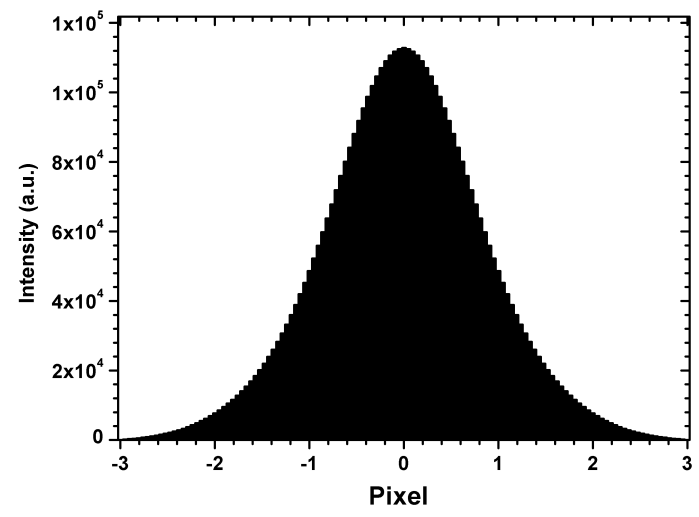

FIG. 4: Point Spread Function PSF (20x over-sampled)

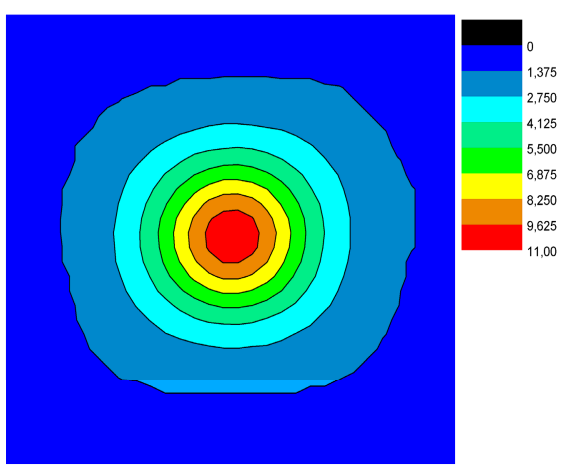

FIG. 3: Interpolated PSF of 11670 events (20x over-sampled).

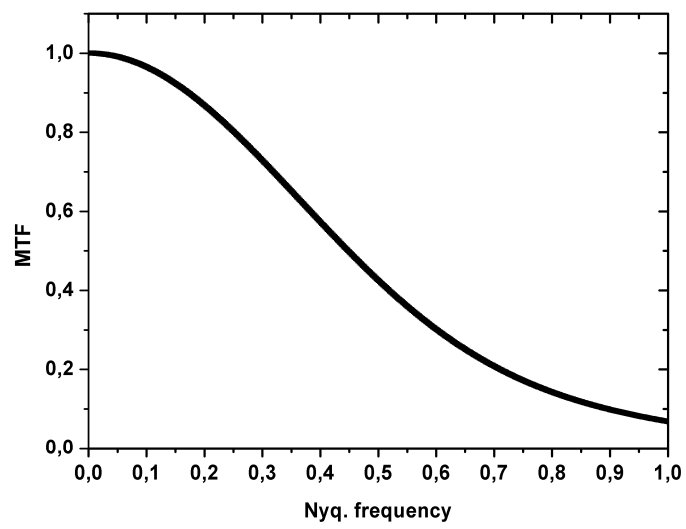

FIG. 5: Modulation Transfer Function MTF (alias-corrected) 